\title{
Use of Antiepileptic Drugs During Pregnancy: Evolving Concepts
}

\author{
Page B. Pennell ${ }^{1}$
}

Published online: 8 August 2016

(C) The American Society for Experimental NeuroTherapeutics, Inc. 2016

\begin{abstract}
Although prenatal exposure to antiepileptic drugs (AEDs) is known to impart relatively higher risks of major congenital malformations, prospective studies have provided refined data that allow us to differentiate the risks of different types and doses of AEDs. As the number of AED prescriptions has dramatically increased in reproductive-aged women with a variety of neuropsychiatric indications, the evolving concepts learned from studies in women with epilepsy can be applied to a much larger group of pregnant women to improve child outcomes while maintaining maternal disease control. In addition to careful selection of the type of medication, the amount of fetal exposure at conception and in the first trimester probably matters across all AEDs. Some AED polytherapy regimens are not associated with a higher risk of malformations, although other outcomes have not yet been formally studied. The individual woman's drug target concentration should be established preconception and maintained during pregnancy, to prevent seizure worsening. Substantial pharmacokinetic changes occur with many of the medications during pregnancy and postpartum, and interindividual variability supports the use of therapeutic drug monitoring for most AEDs. During pregnancy, vigilance and close monitoring should also include intrauterine fetal growth, obstetric complications, and neonatal complications. Breastfeeding can provide additional neurodevelopmental benefit and should be an option for women on AEDs. Knowledge of these key principles enhances our ability to make treatment recommendations with resultant improved maternal and child
\end{abstract}

Page B. Pennell

PPENNELL@BWH.HARVARD.EDU

1 Department of Neurology, Division of Epilepsy, Division of Women's Health, Harvard Medical School, Brigham and Women's Hospital, Boston, MA 02115, USA outcomes. Additional prospective studies are needed to further define the risk-benefit ratio across a variety of medications, dosing strategies, and neuropsychiatric disorders.

Keywords Epilepsy · Antiepileptic drugs · Pregnancy · Breastfeeding $\cdot$ Neuropsychiatric disorders

\section{Introduction and Magnitude of Antiepileptic Drug Use During Pregnancy}

Epilepsy requires continuous treatment during pregnancy with known teratogens. However, significant advances have been made in defining and differentiating risks amongst the various antiepileptic drugs (AEDs), with the potential to improve maternal and child outcomes. Treatment principles are continuing to evolve as new information becomes available, allowing the clinician to better refine treatment choices and dosing strategies during pregnancy.

One aspect that has clearly changed over the last 2 decades is the practice of prescribing AEDs for treatment of other neuropsychiatric disorders, with a major increase in the number of pregnancies at risk owing to prenatal AED exposure. Approximately 1.5 million women with epilepsy (WWE) are of childbearing age in the USA and give birth to approximately 3-5 babies per 1000 born [1]. However, the total number of children in the USA exposed in utero to AEDs is substantially greater with the emergence of AED use for other illnesses, including headache, chronic pain, obesity, mood disorders, and other psychiatric diagnoses. One study estimated that 4.3 million AED prescriptions were written annually to women of childbearing age in the USA [2]. AED use during pregnancy is also substantially higher when considering all neuropsychiatric indications. Prevalence of AED use increased from 15.7 per 1000 deliveries in 2001 to 21.9 per 1000 
deliveries in 2009 , primarily driven by a 5 -fold increase in the newer AEDs [3]. Psychiatric disorders were the most prevalent diagnoses, followed by epilepsy and pain disorders. Of particular concern is the pattern of AED prescriptions in women of childbearing age for neuropsychiatric indications other than epilepsy. Using de-identified data from the National Hospital and Ambulatory Medical Care Surveys (19962007), investigators examined individual prescriptions for adolescent girls and adult women aged 15 to 44 years in the USA [2]. They found that the prevalence of AED prescriptions among women without epilepsy tripled during the study period [10.3 (1996-98) vs 34.9 (2005-07) per 1000 patient visits], and the number of valproate (VPA) prescriptions increased slightly [3.1 (1996-98) vs 3.7 (2005-07) per 1000 patient visits], despite multiple, consistent reports that VPA carries the highest structural and neurodevelopmental teratogenic risks. Of these VPA prescriptions, $83 \%$ were issued to women without epilepsy with $74 \%$ of these for psychiatric diagnoses. The same principles discussed in this article can be applied to treatment during pregnancy of pain, migraine, mood, and other neuropsychiatric disorders with medications that fall under the imprecise label, "antiepileptic drugs."

Treatment of epilepsy during pregnancy can serve as a model for several neuropsychiatric disorders, which are often treated with medications that have a narrow therapeutic index, with lower doses or serum concentrations resulting in uncontrolled maternal disease symptoms and higher doses or serum concentrations resulting in maternal side effects. In addition to these concerns, teratogenic risk increases with the amount of fetal exposure, thus creating a paradigm that medication should be maintained at the lowest possible dose without losing its therapeutic benefit during pregnancy. Epilepsy provides one of the most objective models of this precarious balancing act, as lower serum concentrations of the AEDs often have immediate and measurable consequences of seizure worsening, and higher serum concentrations often results in identifiable side effects. Data from pregnancy registries, case-control studies, population-based studies, and more intensive observational studies of WWE have provided key data that allow us to lower the risk for the developing fetus to rates closer to the general population while maintaining maternal therapeutic benefit.

\section{Relative Risks for Major Congenital Malformations}

In 2009, the Quality Standards Subcommittee and Therapeutics and Technology Assessment Subcommittee of the American Academy of Neurology (AAN) and the American Epilepsy Society (AES) published a 3-part, evidence-based review, "Management issues for women with epilepsy" $[1,4,5]$. Although the major findings of this review continue to provide a foundation for treatment decisions for
WWE during childbearing years, findings from more recently published studies provide further nuances and details that should be incorporated into contemporary treatment and counseling strategies for adolescent and adult WWE.

\section{Major Congenital Malformations}

Major congenital malformations (MCM) are defined as an abnormality of an essential anatomical structure present at birth that interferes significantly with function and/or requires major intervention. The reported MCM rates in the general population vary between $1.6 \%$ and $3.2 \%$, and women with a history of epilepsy but on no AEDs show similar MCM rates. The 2009 AAN/AES practice parameter on "Management issues for women with epilepsy-focus on pregnancy" [1] led to many important conclusions about intrauterine first trimester exposure and risk for MCMs: 1) it is highly probable that VPA exposure has a higher risk of MCMs than carbamazepine (CBZ) and possible higher risk than phenytoin (PHT) or lamotrigine (LTG); 2) compared with untreated WWE, it is probable that VPA as part of polytherapy and possible that VPA as monotherapy contributes to the development of MCM; 3 ) it is probable that AED polytherapy as compared with monotherapy regimens contributes to the development of MCM; 4) CBZ probably does not substantially increase the risk of MCM in the offspring of WWE; and 5) there is probably a relationship between the dose of VPA and LTG and the risk of development of MCMs in the offspring of WWE. The lack of information for many of the other commonly prescribed AEDs is notable at the time of this Practice Parameter.

Since this evidence-based review of the literature, several large prospective pregnancy registries, case-control studies, and population-based studies scattered across different regions of the world have provided valuable information. They reveal a very consistent pattern of amplified risk for the development of MCM in pregnancies exposed to VPA. The registries have also provided updated information on additional AEDs that further refines our ability to lower the teratogenicity risk in WWE.

In 2012, the North American AED Pregnancy Registry (NAAPR) released findings comparing the risk of MCM among infants exposed to different AED monotherapies during the first trimester, as well as to an unexposed reference group [6]. The LTG monotherapy group was chosen as the exposed reference group for the other AEDs because of a low MCM rate and tight confidence intervals [CIs; $2.0 \%$ (95\% CI 1.4-2.8)]. The tables in this article provide detailed information on many of the AEDs with sample size and calculation of CIs for the risk numbers presented. Also, the investigators examined the frequency of specific MCMs for each AED, and reported that VPA was associated with an increased risk of hypospadias, neural tube defects, and cardiovascular malformations; phenobarbital (PB) with an increased risk of cardiovascular 
malformations; and the risk of oral clefts was higher among infants exposed to PB, VPA, and topiramate (TPM), consistent with previous reports. MCM findings should be replicable across studies and across different regions of the world. A systematic review of 21 prospective studies demonstrated a similar pattern of specific malformations, as well as the association of spina bifida with CBZ monotherapy [7].

\section{Dose at Conception Matters for MCM Risk}

The European and International Registry of Antiepileptic Drugs and Pregnancy (EURAP) confirmed that in addition to the type of AED, dose of the AED at conception also affects rates of MCM [8]. MCM rates in pregnancies exposed to CBZ, LTG, VPA, and PB were analyzed by dose at time of conception (not throughout the first trimester or entire pregnancy). The lowest rates of MCMs occurred with LTG $<300 \mathrm{mg}$ /day $(2.0 \%$; $95 \%$ CI 1.19-3.24), and this group was used as the comparator group. Risks of MCMs were higher with VPA and PB at all doses, and with CBZ at $>400 \mathrm{mg} /$ day. The UK and Ireland Epilepsy and Pregnancy Registers published a subsequent report on an even larger number of pregnancies in each medication group and also demonstrated a significant dose effect with VPA- and CBZ-exposed pregnancies, although the dosedependent trend with LTG was not significant [9]. Additionally, the authors reported that the MCM rate for highdose LTG ( $>400 \mathrm{mg} /$ day) appeared lower than the MCM rate for pregnancies exposed to $<600 \mathrm{mg} /$ day of VPA, but, again, this was not significant $(3.4 \%$ vs $5.0 \% ; p=0.31)$.

Although data are not available for all AEDs, the principle that the amount of early fetal exposure matters may be true across the entire medication class of AEDs; pregnancy registries do not yet have the number of enrollments in other AED monotherapy categories to determine this. The finding of an increase in MCM rates with increasing doses for the AEDs studied, even for low MCM-risk AEDs, shifts treatment paradigms. If an individual woman's AED daily dose is on the higher end of the usual dosing range, and she does not have a clear history demonstrating that she needs a high serum concentration for her level of seizure control, attempts should be made to reduce AED doses prior to conception to further reduce the risk of structural teratogenicity. Determining the women's ideal individual target concentration preconception can be a valuable tool for therapeutic drug monitoring during pregnancy (see below).

\section{Polytherapy Rule Reconsidered}

The 2009 AAN/AES practice parameters concluded that "it is probable that AED polytherapy as compared to monotherapy regimens contributes to the development of MCMs". Avoiding polytherapy as a treatment standard in WWE of childbearing age is especially problematic when the clinician is trying to avoid VPA in a woman with a genetic generalized epilepsy syndrome, for example juvenile myoclonic epilepsy. Fortunately, new evidence challenges this treatment paradigm.

Holmes et al. [10] reported on findings from the North American AED Pregnancy Registry that not all AED polytherapy combinations are alike. Focusing on LTG or CBZ as polytherapy, both AEDs had relatively modest rates for MCMs if the polytherapy combination was with any AED other than VPA. Crude odds ratios (OR) were calculated compared to monotherapy with the same AED. The MCM rates were $9.1 \%$ for LTG plus VPA (OR 5.0; $95 \%$ CI $1.5-14.0$ ) but only $2.9 \%$ for LTG with any other AED (OR 1.5; $95 \%$ CI 0.7-3.0); likewise, the risks were $15.4 \%$ for CBZ plus VPA (OR 6.2; $95 \%$ CI 2.0-16.5) and $2.5 \%$ for CBZ plus any other AED (OR 0.8; $95 \%$ CI 0.3-1.9). The UK and Ireland Epilepsy and Pregnancy Register group also reported on MCM rates in levetiracetam (LEV) polytherapy combinations. Although the number of polytherapy patients $(n=367)$ was fairly low with wide CIs and overlapping results, findings suggested that MCM rates were low when LEV was used in combination with LTG $(1.8 \%$; $95 \%$ CI 0.5-6.2 \%), high with VPA (6.9\%; $95 \%$ CI 1.9-22.0\%), and high with CBZ (9.4\%; $95 \%$ CI 4.4-19.0\%). The latter surprising finding highlights that with so many polytherapy combinations to study it could be a long time until enough data are available on many specific polytherapy combinations, never mind at various doses and with various baseline risk factors.

\section{New Data on Commonly Prescribed AEDs Across Neuropsychiatric Disorders}

\section{$T P M$}

Several AED pregnancy registries have reported findings on TPM, an AED prescribed commonly for migraines and weight loss, as well as epilepsy. MCM rates are reported as $4.2 \%$ to $4.9 \%$ for monotherapy use, with an increased risk of cleft lip and cleft palate and hypospadias $[11,12]$. The Australian pregnancy register reported a particularly high rate of MCMs for TPM when used as polytherapy, at $14.1 \%$ with a relative risk of 4.32 (95\% CI 1.57-11.05) compared with no AED exposure [13]. Another major concern is a high rate of small for gestational age (SGA) births following in utero exposure to TPM; the NAAPR reported that $17.9 \%$ of TPMexposed infants were SGA with a relative risk of 2.4 (95\% CI 1.8-22) compared with LTG [14]. In 2011, the Food and Drug Administration made a label change, from a Pregnancy Category $\mathrm{C}$ to $\mathrm{D}$, based on these reports [15].

\section{LEV}

The UK and Ireland Epilepsy and Pregnancy Registers combined results for first trimester exposure to LEV with outcome 
data for 304 monotherapy pregnancies and 367 polytherapy pregnancies [16]. The MCM rate in the LEV monotherapy group was $0.70 \%$ (95\% CI 0.19-2.51) and in the polytherapy group was $5.56 \%$ (95\% CI 3.54-8.56). Findings for specific polytherapy combinations are discussed above. The NAAPR reported on 450 first trimester LEV monotherapy exposures, and the MCM rate was $2.4 \%$ (95\% CI 1.2-4.3) [6].

\section{Gabapentin}

Although gabapentin is widely prescribed for a variety of neuropsychiatric disorders, sparse data are available on its risk during pregnancy. The NAAPR did report a MCM rate of $0.7 \%$ with fairly wide $95 \%$ CIs $(0.02-3.80)$, given that only 145 pregnancies were captured [6]. The prospective AED pregnancy registries are structured to capture WWE better than women with other neuropsychiatric indications. Therefore, it is important to gather data from other study approaches. A prospective cohort study of the teratogen information services and pharmacovigilance centers in Canada, Europe, and Korea reported on 223 gabapentin-exposed women and 223 controls [17]. Rates of MCMs were similar in the 2 groups, ( $4.1 \%$ in the gabapentin group), but the gabapentin group had higher rates of preterm births, low birth weight, and neonatal complications; $38 \%$ were admitted to the neonatal intensive care unit or special care nursery versus only $2 \%$ in the control group. The indications for the gabapentin prescriptions were epilepsy (34\%), pain (43\%), and psychiatric conditions (22\%). Many other medications exposures occurred in these pregnancies, including other AEDs, opiates, and selective serotonin reuptake inhibitors, reflecting the more common prescribing practices seen with gabapentin.

\section{Pregabalin}

Many of the same authors of the gabapentin study reported on pregnancy outcomes following in utero pregabalin exposure [18]. In this multicenter, prospective observational study across 8 participating Teratology Information Services in 7 countries, the prescribing indication for pregabalin was epilepsy in only $3.0 \%$ of the women; the most common indications were pain (neuropathic pain and migraine; $74.3 \%$ ) and psychiatric disorders $(45.1 \%)$, with obvious overlaps. After limiting to first trimester exposures and excluding chromosomal aberration syndromes, $6.0 \%$ of pregabalin-exposed pregnancies versus $2.1 \%$ of control pregnancies had an MCM, generating an OR of 3.0 (95\% CI 1.2-7.9), and CNS-specific malformation rates were higher in the exposed group at $3.2 \%$ versus $0.5 \%$ (OR 6.2; $95 \%$ CI 1.4-28.3). However, all of these cases of MCMs were on other medications and the structural abnormalities reported were relatively vague; none had more specific findings such as neural tube defects. Neonatal complications were assessed in the small number of newborns exposed to pregabalin antenatally until delivery, and 5 of 13 suffered neonatal complications. Important baseline differences between the pregabalin group and the control group included tobacco use (higher in the pregabalin group), other medical conditions, concurrent medications, and gestational age at contact (earlier in the pregabalin group). Unlike use of AEDs for epilepsy, pregabalin was stopped in the majority of these women soon after pregnancy diagnosis. This study provides a signal of concern that MCMs are higher in pregnancies of women on pregabalin with a variety of other factors compared with a control population. However, to differentiate whether this risk is attributable to pregabalin itself, these findings need to be replicated in a larger study and in different populations.

It will be difficult to obtain pure monotherapy data for gabapentin or pregabalin, and especially without concomitant CNS-active medications, as both are commonly prescribed for other neuropsychiatric disorders as part of a cocktail of medications. However, it is erroneous to assume that these medications are benign, even if adverse effects are increased, in part, owing to the concomitant medications or even diseaseassociated risk factors.

\section{Neonatal and Obstetric Complications}

The report of higher neonatal complications in the gabapentin outcomes study increases the awareness of the types of neonatal complications for which to monitor following prenatal exposure to AEDs [17]. Findings from the 2009 AAN/AES practice parameter Update [1] concluded the following : neonates of WWE taking AEDs probably have an increased risk of being SGA of about twice the expected rate. In addition to TPM, elevated prevalence of SGA births has been reported with zonisamide, VPA, and CBZ [14, 19]. A retrospective, cross-sectional study utilizing 2 nationwide population-based data sets reported increased ORs (1.3-1.6) for SGA, low birth weight, and preterm deliveries in women who had seizures during pregnancy compared with women without epilepsy, and the risk of SGA births was still increased when compared with WWE who were seizure-free during pregnancy (OR 1.34; $95 \%$ CI 1.01-1.84). These findings suggest that seizures may directly play a contributory role in adverse neonatal outcomes, although well-designed prospective studies are still needed to untangle the various contributions from seizure types and frequency, and the type, number, and doses of prescribed AEDs.

For obstetric complications, the 2009 AAN/AES practice parameter concluded that for WWE taking AEDs, there is probably no substantially increased risk ( $>2$ times expected) of cesarean delivery or late pregnancy bleeding, and probably no moderately increased risk ( $>1.5$ times expected) of premature contractions or premature labor and delivery. There is possibly a substantially increased risk of premature 
contractions and premature labor and delivery during pregnancy for WWE who smoke. However, newer studies are starting to shed some light on this important topic. A recent retrospective cohort study of pregnant women identified through delivery hospitalization records from the Nationwide Inpatient Sample examined obstetrical outcomes. The study team reported a heightened risk for several obstetric complications during delivery hospitalization if the diagnostic codes included epilepsy, including cesarean section, prolonged hospital stay, pregnancy-related hypertension, hemorrhage, preterm labor, stillbirth, and even an 11.5 OR (95\% CI 8.64-15.19) of death during the delivery hospitalization [20]. The limitations of this study include inability to determine if each complication was related to seizures or AEDs, the inability to determine the type of seizures and epilepsy syndromes, or even verify the diagnosis of epilepsy. However, it does suggest the need to provide attentive care to WWE during delivery hospitalizations. Well-designed, prospective studies should be able to better sort out the various contributory factors to increased obstetrical risks.

\section{Neurodevelopmental Outcomes}

The 2009 AAN practice parameter reported the following conclusions about in utero exposure (throughout the entire pregnancy) and risk for poor cognitive outcomes [1]: 1) cognition is probably not reduced in children of untreated WWE; 2) CBZ probably does not increase poor cognitive outcomes compared with unexposed controls; 3) monotherapy exposure to VPA probably reduces cognitive outcomes; 4) monotherapy exposure to PHT or PB possibly reduces cognitive outcomes; 5) AED polytherapy exposure probably reduces cognitive outcomes compared with AED monotherapy. Since then, several notable reports have contributed to our understanding of the various contributors to adverse neurodevelopmental outcomes and the pattern seen. The Neurodevelopmental Effects of Antiepileptic Drugs study was a prospective, observational, multicenter study in the USA and UK, and assessed the neurodevelopmental effects of in utero exposure to 4 monotherapy groups (CBZ, VPA, PHT, and LTG) [21]. The primary outcome was intelligence quotient (IQ) at 6 years of age, adjusted for maternal IQ, AED type, AED standardized dose, gestational age at birth, and use of periconceptional folate. Primary analysis included 305 mothers and 311 children, with 224 children completing the 6-year follow-up. Multivariate analysis demonstrated that the VPA-exposed children had lower age-6 IQ than CBZ, LTG, or PHT, and they did poorly on several specific measures. High doses of VPA were negatively correlated with IQ, verbal ability, nonverbal ability, memory, and executive function, while the other AEDs did not have a dose effect. Interestingly, mean IQs were higher in the children of mothers who took periconceptional folic acid. This key evidence of a beneficial effect of supplemental folic acid taken prior to and early in pregnancy (in addition to later in pregnancy) in WWE on AEDs supports the recommendation that all women of childbearing age should be encouraged to take supplemental folic acid, especially given the high unplanned pregnancy rate.

The neurodevelopment research group from Liverpool and Manchester also reported that children assessed at 6 years of age who were exposed to VPA in utero had a higher prevalence of neurodevelopmental disorders [22]. Multiple logistic regression analysis revealed that the children born to WWE and exposed to VPA monotherapy and VPA polytherapy were, respectively, 6 times [adjusted OR 6.05; $95 \%$ CI $1.65-24.53(p=0.007)]$ and 10 times [adjusted OR 9.97; $95 \%$ CI 1.82-49.40 $(p=0.005)$ ] more likely to be diagnosed with a neurodevelopmental disorder than controls. Moreover, autism spectrum disorder was the most frequent diagnosis in the VPA-exposed children. A population-based study from Denmark found that in the 508 children exposed to VPA, an absolute risk for autism spectrum disorder of $4.42 \%(95 \% \mathrm{CI}$; 2.59-7.46) (adjusted hazard ratio $2.9 ; 95 \%$ CI 1.7-4.9) and an absolute risk for childhood autism of $2.5 \%$ (95\% CI 1.304.81) (adjusted hazard ratio 5.2; $95 \%$ CI 2.7-10.0) [23]. When restricting the cohort to the children born to WWE, risks were similarly elevated in those children exposed to VPA compared with other AED exposures.

The Liverpool and Manchester Neurodevelopment Group with the UK Epilepsy and Pregnancy Registry reported on the neurodevelopmental outcomes of children exposed in utero to LEV compared with control children and children exposed in utero to VPA [24]. Testing of the children occurred at the age of 36 to 54 months. After adjusting for confounding variables, children exposed to VPA in utero scored lower on measures of gross motor skills, comprehension language abilities, and expressive language abilities than children exposed in utero to LEV. Children exposed to LEV in utero did not differ from the unexposed control children.

Similar concerns for VPA were recently reported by the NAAPR with evaluation of adaptive behavior outcomes of children exposed in utero to LTG, CBZ, or VPA [25]. Even though this study was limited to maternal telephone interviews with use of the Vineland-II Adaptive Behavior Scales for children aged 3 to 6 years of age, significant differences were found between the groups. In utero VPA exposure was associated with dose-dependent adaptive behavior impairments with specific deficits in socialization and motor function, and a relative weakness in communication.

Informed counseling of adolescents and women of childbearing age about use of VPA should include the increased risk for neurodevelopmental disorders, including autism spectrum disorders, as well as increased risk of MCM. Because of these neurodevelopmental adverse effects, on 13 May 2013, a Food and Drug Administration Drug Safety communication 
was released, stating that VPA is contraindicated for migraine prevention in pregnant women and changed from pregnancy category D to category $\mathrm{X}$, although remained category $\mathrm{D}$ for epilepsy or bipolar disorder but should only be prescribed if other medications are not effective [26]. The CMDh (Coordination Group for Mutual Recognition and Decentralized Procedures-Human), a regulatory body representing the European Union member states, released a statement on 21 November 2014 that strengthened the warnings on the use of VPA in women and girls owing to the risk of malformations and developmental problems. The statement included that doctors in the European Union are "now advised not to prescribe VPA for epilepsy or bipolar disorder in pregnant women, in women who can become pregnant or in girls unless other treatments are ineffective or not tolerated. Those for whom the VPA is the only option for epilepsy or bipolar disorder should be advised on the use of effective contraception and treatment should be started and supervised by a doctor experienced in treating these conditions" [27].

Although these warnings seem straightforward, they create a difficult bind when an individual female patient has not responded to other AED therapies. In addition to concern about VPA, it is important to highlight that the neurodevelopmental risks for many of the AEDs are not yet known, and there is concern as stated in the 2009 review [1] that AED polytherapy exposure probably reduces cognitive outcomes compared with AED monotherapy. As the shift continues away from VPA for treatment of epilepsy in women, strengthened by recent reassuring data that some polytherapy combinations may not have a substantially elevated MCM risk, it is likely that AED polytherapy use during pregnancy is increasing [28]. Study of the neurodevelopmental outcomes of these children with AED polytherapy exposure prenatally is essential.

\section{Role of Therapeutic Drug Monitoring in Maintaining Seizure Control During Pregnancy}

The 2009 AAN/AES practice parameter concluded the following: pregnancy probably causes an increase in the clearance and a decrease in the concentration of LTG, PHT, and, to a lesser extent, CBZ, and possibly decreases the level of LEV and the active oxcarbazepine (OXC) metabolite, the monohydroxy derivative. Monitoring of LTG, CBZ, and PHT levels during pregnancy should be considered, and monitoring of LEV and OXC (as monohydroxy derivative) levels may be considered [29]. The magnitude of enhanced clearance of LTG and OXC during pregnancy exceeds that described for many of the older AEDs, owing to elimination via hepatic glucuronidation. This elimination pathway is particularly susceptible to activation during pregnancy, likely owing to the direct effects of rising sex steroid hormone levels. The recommendation to monitor LTG levels is based upon 1 class I study
[30] and 2 class II studies [31, 32], all demonstrating a substantial increase in LTG clearance between pre-pregnancy baseline and the second and third trimesters. Findings from the class I prospective study demonstrated that both LTG free and total clearance were increased during all 3 trimesters, with peaks of $94 \%$ (total) and $89 \%$ (free) in the third trimester. This study also examined therapeutic drug monitoring and seizure frequency, and changes in LTG dosing to avoid postpartum toxicity. The authors reported that seizure frequency significantly increased when the LTG level decreased to $65 \%$ of the preconceptional individualized target LTG concentration, supporting the recommendation to monitor levels of LTG and possibly other AEDs for which the levels decrease during pregnancy. Moreover, the authors demonstrated negligible postpartum toxicity if LTG daily dose was returned to prepregnant doses (or with an additional $50 \mathrm{mg}$ to combat the effects of sleep deprivation) over 10 days postpartum with steady decrements on postpartum days 3, 7, and 10 [30]. Nonadherence to the standard taper schedule was associated with significantly higher risk of experiencing postpartum LTG toxicity (dizziness, imbalance, and blurred or double vision).

The clinical consequences of the gestational-induced pharmacokinetic changes were noted early when the newergeneration AEDs started becoming more commonly prescribed in pregnancy. Specifically, in the prospective EURAP registry, the occurrence of tonic-clonic seizures was associated with OXC monotherapy (OR 5.4; 95 \% CI 1.617.1), and the number or dosage of AEDs were more often increased in pregnancies with monotherapy with LTG (OR $3.8 ; 95 \%$ CI 2.1-6.9) or OXC (OR 3.7; $95 \%$ CI 1.1-12.9) [33]. The major elimination pathway for OXC is also glucuronidation. EURAP followed up these findings with a more recent study comparing seizure control from the first to the second and third trimesters [34]. Preconceptional baseline seizure frequency was not available, nor was information on AED blood levels. The authors reported that compared with other AED monotherapies, pregnant women on LTG monotherapy were less likely to be seizure-free (58.2\%), had more generalized tonic-clonic seizures $(21 \%)$, and had a greater likelihood of deterioration in seizure control from the first trimester to later trimesters (19.9\%), and were more likely to require an increase in drug load (47.7 \%) [34]. However, the mean dose increase from the first to third trimesters was only $26 \%$ for LTG, and the authors suggested that a more proactive therapeutic drug monitoring approach should be considered, especially for those on LTG.

These prior studies of LTG clearance during pregnancy demonstrated substantial interindividual variability in the magnitude of the enhanced LTG clearance, and a more recent formal pharmacokinetic analysis utilizing a population-based, nonlinear, mixed-effects model demonstrated 2 subpopulations [35]. The majority of the women (77\%) displayed a marked increase in LTG clearance, whereas $23 \%$ had a 
minimal increase in LTG clearance from baseline, with a 10fold higher rate in the larger subpopulation group. To put this in practical clinical terms, the estimated rate of increase in LTG clearance for this main population predicts $76 \%$, $153 \%$, and $219 \%$ increase from baseline, nonpregnant clearance by the end of the first, second, and third trimesters, respectively; the smaller subpopulation is predicted to have lower degree of increase in LTG clearance of $7.5 \%, 15 \%$, and $21 \%$ by the end of each trimester. The authors of this study suggest that genotypic variation may affect activity and induction of UGT1A4; however, more studies are needed to clarify this. The substantial differences between these 2 subpopulations argues for therapeutic drug monitoring during pregnancy, at least until we have reliable genetic testing to determine which subpopulation an individual woman will be in. Additionally, this formal pharmacokinetic modeling study clarified that the LTG clearance declined in an exponential manner with an expected return to baseline clearance 3 weeks after delivery, and thus it may be more appropriate to return to pre-pregnant doses (or a slightly higher dose) over $2-3$ weeks rather than 10 days.

Although the direct clinical consequences of accelerated clearance of LTG during pregnancy without use of dosage adjustments has been well demonstrated, little is published about other AEDs, including common ones that are known to also undergo major pharmacokinetic alterations during pregnancy. For example, renal clearance increases substantially during pregnancy, and small studies demonstrate that LEV levels decrease by $40 \%$ to $62 \%$ during the second and third trimesters [36-39]. A more detailed analysis of 12 pregnancies demonstrated an even greater change in clearance of LEV from nonpregnant baseline to the third trimester, with a mean \pm SD increase from $124.7 \pm 57.91 /$ day to $427.3 \pm 211.3$ $(p<0.0001)$, an increase of $242 \%$ [37]. However, the effects on seizure control are less clear from these studies.

One group of researchers investigated if the " $65 \%$ rule" determined with LTG held true with other AEDs. In other words, when the AED serum concentration fell to $<65 \%$ of preconception baseline, did seizures worsen? Using a retrospective analysis of clinic patients at a single epilepsy center with 115 pregnancies in 95 women, they reported that significant changes in clearance occurred with LTG and LEV with average peak clearance increases of $191 \%$ and $207 \%$, respectively, above nonpregnant baseline [40]. Marked variance was seen across women and even across repeat pregnancies in the same women, reinforcing the value of therapeutic drug monitoring even for subsequent pregnancies. Despite increasing doses across most AEDs, seizures still increased in $38.4 \%$ of women during pregnancy, and seizure deterioration was significantly more likely in patients during the second trimester when the AED concentration fell to $<65 \%$ of preconception baseline (after controlling for baseline seizure occurrence). Other factors associated with seizure deterioration during pregnancy were the presence of seizures in the 12 months prior to conception and focal seizure types, similar to reports from the EURAP study [34].

These studies highlight the importance of therapeutic drug monitoring during pregnancy to help prevent seizure deterioration in woman on a variety of AEDs and support recommendations by several experts on adjusting AED dosing during pregnancy accordingly [41-43]. One finding that stood out for women on CBZ in these prior studies was that they had a very low rate of worsened seizure control $(0-14.6 \%)$ and were less likely to have dosage adjustments during pregnancy $[34,40]$. A more recent analysis helped to clarify the pharmacokinetic changes of CBZ during pregnancy compared with the 2009 AAN/AES pregnancy parameter update [29], and findings provide an explanation for these observations. This prospective study measured total and free $\mathrm{CBZ}$ and $\mathrm{CBZ}$-epoxide (CBZ-EPO) levels in 15 pregnancies (in 12 women) from nonpregnant baseline and each trimester and seizure frequency [44]. No significant changes occurred in the clearance of total and free CBZ or CBZ-EPO throughout the pregnancy compared with nonpregnant baseline. The free fraction of CBZ increased from 0.23 at baseline to a maximum of 0.32 in the third trimester $(p=0.008)$, thus potentially providing additional seizure protection. In the six women on CBZ monotherapy with adequate seizure diaries and blood sampling, seizure worsening did not correspond to a ratio to baseline concentration of $<0.65$ for total or free CBZ or CBZEPO. The authors concluded that for focal-onset seizures when AED blood levels are not readily available, CBZ may be a particularly good choice as it is reasonable to not perform therapeutic drug monitoring during pregnancy and because of its relatively low structural teratogenic risk $[6,45]$, and the normal neurocognitive profiles of the children following prenatal exposure [21]. Gestational-induced pharmacokinetic data are lacking for many of the newer AEDs (e.g., pregabalin, lacosamide, eslicarbazepine acetate, rufinamide, clobazam), in part because prescriptions in pregnant women are often delayed until some teratogenic safety data are available.

\section{Establishing Individual AED Target Concentrations}

The findings reported by EURAP that the dose at conception is important for all AEDs studied [8], suggest that a woman's preconception dose should be scrutinized prior to pregnancy and reduced if possible based upon her seizure history and personal characteristics. It is helpful to measure baseline serum concentration in the nonpregnant state, and use that for guidance if she has good seizure control without side effects, and if it is thought that her dose cannot be lowered further. However, if she is on a concomitant hormonal contraceptive that includes an estrogen, then her dose can often be adjusted lower when the hormonal contraceptive is stopped if she is on LTG, VPA, and probably OXC. LTG clearance is increased 


\section{Teratogenic Risk Profiles of Antiepleptic Drugs}

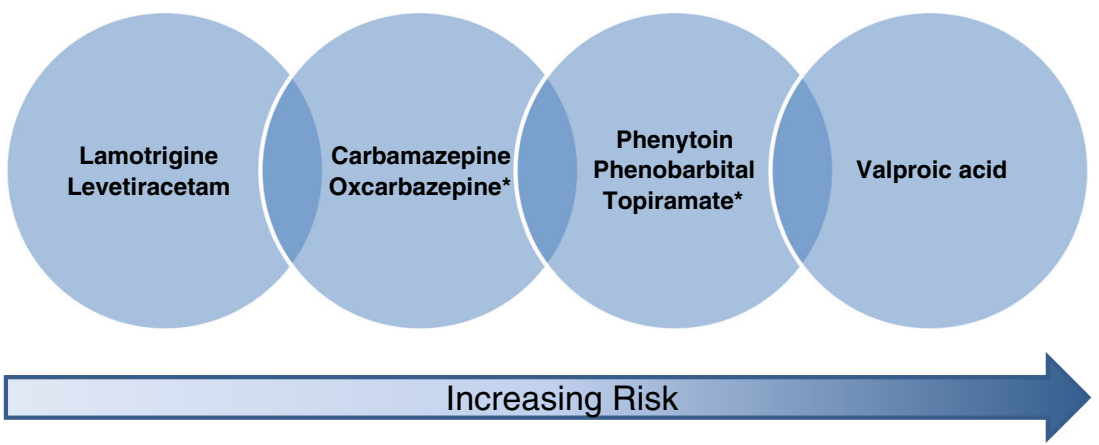

Fig. 1 Summary of relative teratogenic risk profiles of antiepileptic drugs, based on available data at the time of writing. The risk profiles include data about major congenital malformations, fetal growth, and neurodevelopmental outcomes when available, with consideration of the range of relative risks reported from multiple studies, number of patients studied, and confidence intervals. * Neurodevelopmental outcomes are not yet known

breastfeeding is associated with a reduced risk of type 2 diabetes mellitus, breast cancer, ovarian cancer, and maternal postpartum depression $[52,53]$. Additionally, many studies actually demonstrate a positive association of breastfeeding with improved cognitive abilities [54-56]. Fortunately, evidence is now available in epilepsy mother-child pairs that the beneficial effects of breastfeeding are still realized in this population. The Neurodevelopmental Effects of Antiepileptic Drugs study compared the 6-year-old neuropsychometric results of the children who were breastfed versus those who were not. The IQ of the children was related to breastfeeding, with adjusted IQ 4 (95\% CI 0-8) points higher for breastfeeding, and the adjusted verbal index was 4 (95\% CI 0-7) points higher [57]. The other health benefits for the child and for the mother are also likely, although not yet studied formally.

The benefits of breastfeeding are believed to outweigh the small risk of adverse effects of AEDs. This recommendation needs to be balanced with consideration of minimizing sleep disruption. A common compromise is to elicit the help of family members and friends to provide 1 or 2 bottle feedings per $24 \mathrm{~h}$ of either formula or pumped breast milk to allow the mother at least one 4 to 6-h stretch of uninterrupted sleep. Breastfeeding should be supported, if not encouraged, in WWE.

\section{Conclusion}

AEDs are commonly prescribed to women of childbearing age for a variety of neuropsychiatric indications. Most of our data on risks of the AEDs during pregnancy come from studies in WWE, and highlight the importance of maintaining maternal disease control while minimizing prenatal AED exposure. Data continue to accumulate that some AEDs carry a higher structural and neurodevelopmental teratogenic risk than others (e.g., 
VPA), while data are still lacking for many AEDs. Figure 1 summarizes my interpretation of the available data at the time of writing. The risk profiles include data about major congenital malformations, fetal growth, and neurodevelopmental outcomes when available, with consideration of the range of relative risks reported from multiple studies, number of patients studied, and CIs. The AEDs not included do not have enough monotherapy data reported to be included. These risk profiles will likely change as new data becomes available.

Evolving concepts since the 2009 3-part, evidence-based AAN/AES practice parameters on the management issues for WWE with a focus on pregnancy include the following during the preconception, pregnancy, and postpartum stages. Evidence is now available in WWE that supplemental folic acid beginning prior to pregnancy improves neurodevelopmental outcomes of the children, the individual's target concentration should be established, and the dose lowered if possible prior to conception, especially after discontinuation of estrogencontaining contraceptive hormones if on LTG, VPA, and probably OXC. Several polytherapy combinations are reported to have relatively low risks for major congenital malformations, although data on fetal growth, obstetric and neonatal outcomes, and long-term neurodevelopmental outcomes are lacking. During pregnancy, monthly AED levels should be obtained for therapeutic drug monitoring to maintain the nonpregnant individual target concentration for most AEDs studied with the possible exception of CBZ; doses should be adjusted for seizures, side effects, and to prevent the ratio to target concentration from decreasing to $65 \%$ or lower. Postpartum, breastfeeding should be encouraged, similar to women without neuropsychiatric disease and not on medications for the known multiple benefits, but with supplementation with bottle feeding to prevent extreme sleep deprivation. AEDs should be adjusted back to preconception doses or slightly higher over 2 weeks to 3 months, depending on the AED. These same evolving concepts and principles can be applied to women on AEDs for other neuropsychiatric indications that require chronic, daily dosing during pregnancy and postpartum.

Acknowledgments Preparation of this paper was supported, in part, by National Institutes of Health (National Institute of Neurological Disorders and Stroke, National Institute of Child Health and Human Development) \#2U01-NS038455.

Required Author Forms Disclosure forms provided by the authors are available with the online version of this article.

\section{References}

1. Harden CL, Meador KJ, Pennell PB, et al. Management issues for women with epilepsy-focus on pregnancy (an evidence-based review): II. Teratogenesis and perinatal outcomes: Report of the Quality Standards Subcommittee and Therapeutics and Technology
Subcommittee of the American Academy of Neurology and the American Epilepsy Society. Epilepsia 2009;50:1237-1246.

2. Adedinsewo DA, Thurman DJ, Luo YH, Williamson RS, Odewole OA, Oakley GP, Jr. Valproate prescriptions for nonepilepsy disorders in reproductive-age women. Birth Defects Res A Clin Mol Teratol 2013;97:403-408.

3. Bobo WV, Davis RL, Toh S, et al. Trends in the use of antiepileptic drugs among pregnant women in the US, 2001-2007: a medication exposure in pregnancy risk evaluation program study. Paediatr Perinat Epidemiol 2012;26:578-588.

4. Harden CL, Hopp J, Ting TY, et al. Management issues for women with epilepsy - focus on pregnancy (an evidence-based review): I. Obstetrical complications and change in seizure frequency: Report of the Quality Standards Subcommittee and Therapeutics and Technology Assessment Subcommittee of the American Academy of Neurology and the American Epilepsy Society. Epilepsia 2009;50:1229-1236.

5. Harden CL, Pennell PB, Koppel BS, et al. Management issues for women with epilepsy - focus on pregnancy (an evidence-based review): III. Vitamin K, folic acid, blood levels, and breast-feeding: Report of the Quality Standards Subcommittee and Therapeutics and Technology Assessment Subcommittee of the American Academy of Neurology and the American Epilepsy Society. Epilepsia 2009;50:1247-1255.

6. Hernandez-Diaz S, Smith CR, Shen A, et al. Comparative safety of antiepileptic drugs during pregnancy. Neurology 2012;78:1692-1699.

7. Tomson T, Battino D, Bonizzoni E, et al. EURAP: an international registry of antiepileptic drugs and pregnancy. Epilepsia 2004;45: 1463-1464.

8. Tomson T, Battino D, Bonizzoni E, et al. Dose-dependent risk of malformations with antiepileptic drugs: an analysis of data from the EURAP epilepsy and pregnancy registry. Lancet Neurol 2011;10: 609-617.

9. Campbell E, Kennedy F, Russell A, et al. Malformation risks of antiepileptic drug monotherapies in pregnancy: updated results from the UK and Ireland Epilepsy and Pregnancy Registers. J Neurol Neurosurg Psychiatry 2014;85:1029-1034.

10. Holmes LB, Mittendorf R, Shen A, Smith CR, Hernandez-Diaz S. Fetal effects of anticonvulsant polytherapies: different risks from different drug combinations. Arch Neurol 2011;68:1275-1281.

11. Hunt S, Russell A, Smithson WH, et al. Topiramate in pregnancy: preliminary experience from the UK Epilepsy and Pregnancy Register. Neurology 2008;71:272-276.

12. Margulis AV, Mitchell AA, Gilboa SM, et al. Use of topiramate in pregnancy and risk of oral clefts. Am J Obstet Gynecol 2012;207:405.

13. Vajda FJ, O'Brien TJ, Lander CM, Graham J, Eadie MJ. The teratogenicity of the newer antiepileptic drugs - an update. Acta Neurol Scand 2014;130:234-238.

14. Hernandez-Diaz S, Mittendorf R, Smith CR, Hauser WA, Yerby M, Holmes LB. Association between topiramate and zonisamide use during pregnancy and low birth weight. Obstet Gynecol 2014;123:21-28.

15. US Food and Drug Administration. Safety Label Changes 2011. Available at: http://www.fda.gov/Safety/MedWatch/Safety Information/ucm249643.htm. Accessed ??.

16. Mawhinney E, Craig J, Morrow J, et al. Levetiracetam in pregnancy: results from the UK and Ireland epilepsy and pregnancy registers. Neurology 2013;80:400-405.

17. Fujii H, Goel A, Bernard N, et al. Pregnancy outcomes following gabapentin use: results of a prospective comparative cohort study. Neurology 2013;80:1565-1570.

18. Winterfeld U, Merlob P, Baud D, et al. Pregnancy outcome following maternal exposure to pregabalin may call for concern. Neurology 2016;86:2251-2257.

19. Pennell PB, Klein AM, Browning N, et al. Differential effects of antiepileptic drugs on neonatal outcomes. Epilepsy Behav 2012;24: 449-456. 
20. MacDonald SC, Bateman BT, McElrath TF, Hernandez-Diaz S. Mortality and morbidity during delivery hospitalization among pregnant women with epilepsy in the United States. JAMA Neurol 2015;72:981-988.

21. Meador KJ, Baker GA, Browning N, et al. Fetal antiepileptic drug exposure and cognitive outcomes at age 6 years (NEAD study): a prospective observational study. Lancet Neurol 2013;12:244-252.

22. Bromley RL, Mawer GE, Briggs M, et al. The prevalence of neurodevelopmental disorders in children prenatally exposed to antiepileptic drugs. J Neurol Neurosurg Psychiatry 2013;84:637-643.

23. Christensen J, Gronborg TK, Sorensen MJ, et al. Prenatal valproate exposure and risk of autism spectrum disorders and childhood autism. JAMA 2013;309:1696-1703.

24. Shallcross R, Bromley RL, Cheyne CP, et al. In utero exposure to levetiracetam vs valproate: development and language at 3 years of age. Neurology 2014;82:213-221.

25. Deshmukh U, Adams J, Macklin EA, et al. Behavioral outcomes in children exposed prenatally to lamotrigine, valproate, or carbamazepine. Neurotoxicol Teratol 2016;54:5-14.

26. US Food and Drug Administration. FDA Drug Safety Communication: valproate anti-seizure products contraindicated for migraine prevention in pregnant women due to decreased IQ scores in exposed children. Available at: http://www.fda. gov/drugs/drugsafety/ucm350684.htm. Accessed ??

27. European Medicines Agency. CMDh agrees to strengthen warnings on the use of valproate medicines in women and girls 2014 (updated November 21, 2014). Available at: http://www.ema.europa. eu/docs/en GB/document library/Press release/2014/11 /WC500177638.pdf. Accessed ??

28. Pennell PB MK, May RC, French J, et al.; MONEAD Investigator Group. Antiepileptic drug prescribing patterns in women with epilepsy during pregnancy: findings from the MONEAD study. 2015 American Epilepsy Society Annual Meeting; 20152015.

29. Harden CL, Pennell PB, Koppel BS, et al. Practice Parameter Update: management issues for women with epilepsy-focus on pregnancy (an evidence-based review): vitamin K, folic acid, blood levels, and breastfeeding. Neurology 2009;73:142-149.

30. Pennell PB, Peng L, Newport DJ, et al. Lamotrigine in pregnancy: clearance, therapeutic drug monitoring, and seizure frequency. Neurology 2008;70:2130-2136.

31. Pennell PB, Newport DJ, Stowe ZN, Helmers SL, Montgomery JQ, Henry TR. The impact of pregnancy and childbirth on the metabolism of lamotrigine. Neurology 2004;62:292-295.

32. Tran TA, Leppik IE, Blesi K, Sathanandan ST, Remmel R. Lamotrigine clearance during pregnancy. Neurology 2002;59:251-255.

33. EURAP Study Group. Seizure control and treatment in pregnancy: observations from the EURAP epilepsy pregnancy registry. Neurology 2006;66:354-360.

34. Battino D, Tomson T, Bonizzoni E, et al. Seizure control and treatment changes in pregnancy: observations from the EURAP epilepsy pregnancy registry. Epilepsia 2013;54:1621-1627.

35. Polepally AR, Pennell PB, Brundage RC, et al. Model-based lamotrigine clearance changes during pregnancy: clinical implication. Ann Clin Transl Neurol 2014;1:99-106.

36. Westin AA, Reimers A, Helde G, Nakken KO, Brodtkorb E. Serum concentration/dose ratio of levetiracetam before, during and after pregnancy. Seizure 2008;17:192-198.

37. Tomson T, Palm R, Kallen K, et al. Pharmacokinetics of levetiracetam during pregnancy, delivery, in the neonatal period, and lactation. Epilepsia 2007;48:1111-1116.

38. Tomson T, Battino D. Pharmacokinetics and therapeutic drug monitoring of newer antiepileptic drugs during pregnancy and the puerperium. Clin Pharmacokinet 2007;46:209-219.
39. Lopez-Fraile IP, Cid AO, Juste AO, Modrego PJ. Levetiracetam plasma level monitoring during pregnancy, delivery, and postpartum: clinical and outcome implications. Epilepsy Behav 2009;15: 372-375.

40. Reisinger TL, Newman M, Loring DW, Pennell PB, Meador KJ. Antiepileptic drug clearance and seizure frequency during pregnancy in women with epilepsy. Epilepsy Behav 2013;29:13-18.

41. Tomson T, Landmark CJ, Battino D. Antiepileptic drug treatment in pregnancy: changes in drug disposition and their clinical implications. Epilepsia 2013;54:405-414.

42. Patsalos PN, Berry DJ, Bourgeois BF, et al. Antiepileptic drugs — best practice guidelines for therapeutic drug monitoring: a position paper by the subcommission on therapeutic drug monitoring, ILAE Commission on Therapeutic Strategies. Epilepsia 2008;49:1239-1276.

43. Harden CL, Pennell PB, Koppel BS, et al. Practice parameter update: management issues for women with epilepsy-focus on pregnancy (an evidence-based review): vitamin K, folic acid, blood levels, and breastfeeding: report of the Quality Standards Subcommittee and Therapeutics and Technology Assessment Subcommittee of the American Academy of Neurology and American Epilepsy Society. Neurology 2009;73:142-149.

44. Johnson EL, Stowe ZN, Ritchie JC, et al. Carbamazepine clearance and seizure stability during pregnancy. Epilepsy Behav 2014;33:49-53.

45. Tomson T, Battino D. Teratogenic effects of antiepileptic drugs. Lancet Neurol 2012;11:803-813.

46. Sabers A, Ohman I, Christensen J, Tomson T. Oral contraceptives reduce lamotrigine plasma levels. Neurology 2003;61:570-571.

47. Ohman I, Luef G, Tomson T. Effects of pregnancy and contraception on lamotrigine disposition: new insights through analysis of lamotrigine metabolites. Seizure 2008;17:199-202.

48. Reimers A, Helde G, Brodtkorb E. Ethinyl estradiol, not progestogens, reduces lamotrigine serum concentrations. Epilepsia 2005;46: 1414-1417.

49. Wegner I, Wilhelm AJ, Lambrechts DA, Sander JW, Lindhout D. Effect of oral contraceptives on lamotrigine levels depends on comedication. Acta Neurol Scand 2014;129:393-398.

50. Wegner I, Edelbroek PM, Bulk S, Lindhout D. Lamotrigine kinetics within the menstrual cycle, after menopause, and with oral contraceptives. Neurology 2009;73:1388-1393.

51. Herzog AG, Blum AS, Farina EL, et al. Valproate and lamotrigine level variation with menstrual cycle phase and oral contraceptive use. Neurology 2009;72:911-914.

52. Ip S, Chung M, Raman G, Trikalinos TA, Lau J. A summary of the Agency for Healthcare Research and Quality's evidence report on breastfeeding in developed countries. Breastfeed Med 2009;4(Suppl. 1):S17-S30.

53. Figueiredo B, Dias CC, Brandao S, Canario C, Nunes-Costa R. Breastfeeding and postpartum depression: state of the art review. $\mathrm{J}$ Pediatr (Rio J) 2013;89:332-338.

54. Bernard JY, De Agostini M, Forhan A, et al. Breastfeeding duration and cognitive development at 2 and 3 years of age in the EDEN mother-child cohort. J Pediatr 2013;163:36-42.

55. Belfort MB, Rifas-Shiman SL, Kleinman KP, et al. Infant feeding and childhood cognition at ages 3 and 7 years: Effects of breastfeeding duration and exclusivity. JAMA Pediatr 2013;167: 836-844.

56. Julvez J, Guxens M, Carsin AE, et al. A cohort study on full breastfeeding and child neuropsychological development: the role of maternal social, psychological, and nutritional factors. Dev Med Child Neurol 2014;56:148-156.

57. Meador KJ, Baker GA, Browning N, et al. Breastfeeding in children of women taking antiepileptic drugs: cognitive outcomes at age 6 years. JAMA Pediatr 2014;168:729-736. 